\title{
$X V$. On the limits upon the earth's surface within which an occultation of a star or planet by the moon is visible
}

\author{
J.W. Lubbock Esq. F.R.S.
}

To cite this article: J.W. Lubbock Esq. F.R.S. (1831) XV. On the limits upon the earth's surface within which an occultation of a star or planet by the moon is visible, Philosophical Magazine Series 2, 9:50, 90-92, DOI: 10.1080/14786443108647547

To link to this article: http://dx.doi.org/10.1080/14786443108647547

曲 Published online: 01 Jun 2009.

Submit your article to this journal $[\pi$

Џll Article views: 2

Q View related articles $₫$ 
Berwick Hill near Mason Dinnington, and Heddon-on-theWall, and another traverses the country to the westward, from the sea-coast in the vicinity of Howick and Warkworth by the Helm-on-the-Hill, Netherwitton, Roadley, Shaftoe Crags near Wallington, and Stamfordham. Basalt being evidently the production of fire, and pervading in an irregular manner rocks of almost every age, does not come within the scope of these remarks; so that the similarity of organic remains has at length become the geologist's chief guide and reliance, in proving or endeavouring to prove the identity of formations; but if I mistake not these exuviæ will not always warrant the conclusions drawn from their presence.

XV. On the Limits upon the Earth's Surface within which an Occultation of a Star or Planet by the Moon is visible. By J. W. Luввоск, Esq. F.R.S.*

A STAR is occulted at all those points which coincide at
any instant with the intersection of a cone circumscribing the moon, and whose apex coincides with the star in question and the earth's surface; and the star will be just occulted, or appear to graze the moon's edge at those points which coincide with the intersection of a plane perpendicular to the moon's orbit, and passing through the star and the centre of the moon, with the earth's surface. In order, therefore, to determine the limits within which the occultation is visible, it is necessary to find the equation of this cone and plane.

On account of the small diameter of the moon and the great distance of the star or planet, the cone in question may be considered as a cylinder whose axis coincides with the line joining the centres of the moon and the star, which axis is parallel to a line joining the centre of the earth and the star.

Let $\mathrm{F}(x, y, z)=0, \mathrm{~F}^{\prime}(x, y, z)=0$, be the equations to any curve, $x=a z+x, y=b z+y$, the equations to any straight line, the equation to the cylinder whose axis coincides with the line in question and has the curve $\{\mathrm{F}(x, y, z)=0$ $\left.\mathbf{F}^{\prime}(x, y, z)=0\right\}$ for its base, may be found by eliminating $x, y, x$ between the four equations $\left\{\mathrm{F}(x, y, z)=0, \mathrm{~F}^{\prime}(x, y, z)=0\right.$ $x=a z+x_{1}$ and $\left.y=b z+y_{1}\right\}$ and then putting for $x_{1}$ and $y_{1}$ their values $x-a z$ and $y-b z$ in the resulting equation. The following method however in some cases is perhaps more simple.

Let $x^{18}+y^{12}=r^{8}$ be the equation to the cylinder in ques-

- Communicated by the Author. 
tion referred to coordinate axes which have their origin at the moon's centre; the axis $z$ of course coinciding with the line which joins the centres of the moon and the star, and $r$ being the radius of the moon.

Let $x_{1}, y_{1}, z_{1}$, be the coordinates of the centre of the moon referred to the centre of the earth, the plane $x y$ coinciding with the equator, $x_{2}, y_{2}, z_{2}$ the coordinates of the star referred to the same axes, and let

$$
\begin{aligned}
& x^{\prime}=a\left(x-x_{1}\right)+b\left(y-y_{1}\right)+c\left(z-z_{1}\right) \\
& y^{\prime}=a^{\prime}\left(x-x_{1}\right)+b^{\prime}\left(y-y_{1}\right)+c^{\prime}\left(z-z_{1}\right) \\
& z^{\prime}=a^{\prime \prime}\left(x-x_{1}\right)+b^{\prime \prime}\left(y-y_{1}\right)+c^{\prime \prime}\left(z-z_{1}\right)
\end{aligned}
$$

The equation to the cylinder referred to the axes $x, y, z$ is

$$
\begin{gathered}
\left\{a\left(x-x_{1}\right)+b\left(y-y_{1}\right)+c\left(z-z_{1}\right)\right\}^{2} \\
+\left\{\left(a^{\prime}\left(x-x_{1}\right)+b^{\prime}\left(y-y_{1}\right)+c^{\prime}\left(z-z_{1}\right)\right\}^{2}=r^{2}\right.
\end{gathered}
$$

The equation to the plane $x^{\prime} y^{\prime}$ is

$$
a^{\prime \prime}\left(x-x_{1}\right)+b^{\prime \prime}\left(y-y_{1}\right)+c^{\prime \prime}\left(z-z_{1}\right)=0 \text {. }
$$

If $\alpha_{1}$ and $\delta_{1}$ denote the right ascension and declination of the moon, and $\alpha_{2}, \delta_{2}$ of the star, since the plane $x^{\prime} y^{\prime}$ is perpendicular to a line joining the centre of the moon and star, it is easy to show that

$$
a^{\prime \prime}=\cos \alpha_{2} \cos \delta_{2}, b^{\prime \prime}=\sin \alpha_{2} \cos \delta_{2}, c^{\prime \prime}=\sin \delta_{2} \text {. }
$$

The following well-known equations of condition obtain between the quantities $a, b, c, a^{\prime}, b^{\prime}, c^{\prime}, a^{\prime \prime}, b^{\prime \prime}, c^{\prime \prime}$ :

$$
\begin{aligned}
& a a^{\prime}+b b^{\prime}+c c^{\prime}=0 \\
& a a^{\prime \prime}+b b^{\prime \prime}+c c^{\prime \prime}=0 \\
& a^{\prime} a^{\prime \prime}+b^{\prime} b^{\prime \prime}+c^{\prime} c^{\prime \prime}=0 \\
& a^{2}+b^{2}+c^{2}=1 \\
& a^{\prime 2}+b^{\prime 2}+c^{\prime 2}=1 \\
& a^{\prime \prime 2}+b^{\prime \prime 2}+c^{\prime \prime 2}=1
\end{aligned}
$$

Since these equations are more than are necessary to determine these quantities, we may suppose one of them as $c=0$, and then it is easy to show that

$$
\begin{array}{ll}
a=\sin \alpha_{2}, & b=-\cos \alpha_{2} \\
a^{\prime}=\cos \alpha_{2} \sin \delta_{2}, & b^{\prime}=\sin \alpha_{2} \sin \delta_{2}, c^{\prime}=-\cos \delta_{2},
\end{array}
$$

and the equation to the cylinder becomes

$$
\begin{gathered}
\left\{\left(x-x_{1}\right) \sin \alpha_{2}-\left(y-y_{1}\right) \cos \alpha_{2}\right\}^{2} \\
+\left\{\left(x-x_{1}\right) \sin \delta_{2} \cos \alpha_{2}+\left(y-y_{1}\right) \sin \alpha_{2} \sin \delta_{2}\right. \\
\\
\left.-\left(z-z_{1}\right) \cos \delta_{2}\right\}^{2}=r^{2}
\end{gathered}
$$

If $x=R \cos \phi \cos \alpha_{3}, y=R \cos \varphi \sin \alpha_{3}, z=R \sin \ddagger$, $\phi$ being the geographical latitude of a point on the earth's surface, and if II be the moon's horizontal parallax, $\Delta$ her apparent semidiameter, 


$$
\begin{aligned}
& \left\{\cos \delta_{1} \sin \left(\alpha_{2}-\alpha_{1}\right)-\Pi \cos \phi \sin \left(\alpha_{2}-\alpha_{3}\right)\right\}^{2} \\
+ & \left\{\cos \delta \sin \delta_{2} \cos \left(\alpha_{2}-\alpha_{1}\right)-\sin \delta_{1} \cos \delta_{2}\right. \\
& -\Pi\left(\cos \phi \sin \delta_{2} \cos \left(\alpha_{2}-\alpha_{3}\right)-\sin \phi \cos \delta_{2}\right\}^{2}=\Delta^{8}
\end{aligned}
$$

Let $\omega$ be the inclination of the moon's orbit to the equator, $\nu$ the right ascension of its intersection with the equator; then the equation to her orbit is

$$
x \sin \omega \sin y-y \sin \omega \cos y+z \cos \omega=0
$$

The equation to a plane passing through the star and the centre of the moon and perpendicular to this plane is

$$
\begin{aligned}
& \left(x-x_{1}\right)\left(\cos \omega \cos \delta_{2} \sin \alpha_{2}+\sin \delta_{2} \sin \omega \cos \nu\right) \\
+ & \left(y-y_{1}\right)\left(\sin \omega \sin \nu \sin \delta_{2}-\cos \delta_{2} \cos \alpha_{2} \cos \omega\right) \\
- & \left(z-z_{1}\right)\left(\sin \omega \cos \delta_{2} \cos \left(\alpha_{2}-\nu\right)=0,\right.
\end{aligned}
$$

and putting $R \cos \phi \cos \alpha_{3}$ for $x, R \cos \phi \sin \alpha_{3}$ for $y$, and $R \sin \varphi$ for $z$,

$$
\begin{gathered}
\Pi\left\{\cos \phi \sin \delta_{2} \sin \omega \cos \left(\nu-\alpha_{3}\right)+\cos \phi \cos \delta_{2} \cos \omega \sin \left(\alpha_{2}-\alpha_{3}\right)\right. \\
\left.-\sin \phi \cos \delta_{2} \sin \omega \cos \left(\alpha_{2}-\nu\right)\right\}
\end{gathered}
$$

$$
-\cos \delta_{1} \sin \delta_{2} \sin \omega \cos \left(\nu-\alpha_{1}\right)-\cos \delta_{1} \cos \delta_{2} \cos \omega \sin \left(\alpha_{2}-\alpha_{1}\right)
$$$$
+\sin \delta_{1} \cos \delta_{2} \sin \omega \cos \left(\alpha_{2}-\nu\right)=0 \text {, }
$$

the geographical longitude east of the point corresponding to $\alpha_{3}=\alpha_{3}$ - the sidereal time at the place from which longitudes are reckoned.

Equation (1) may be considered as the equation of a cone having its apex at the earth's centre, the variables being $\varphi$ and $\alpha_{3}$. This determines the points on the earth's surface for which the star is occulted at any instant; the intersection of this cone with the plane, Equation (2), determines the points at which the star is just occulted or appears to graze the edge of the moon. The mathematical solution here given comprehends also those points on the opposite hemisphere of the earth to the moon, which are intersected by the cylinder and plane, and which must obviously be excluded.

XVI. Tables of the Decimal Parts of a Day and an Hour. By A Correspon Dent.

To the Editors of the Philosophical Magazine and Annals. Gentlemen,

A $\mathrm{S}$ I see that in a former Number of your Journal you $A$ have commenced a publication of correct 'Tables requisite in practical astronomy, I forward two of the same nature, in the hope that other correspondents may be induced, like myself, to follow so useful an example.

December 10, 1830. 